\title{
The Influence of Mindfulness and Positive Psychological Capital on Psychological Burnout of Middle-Aged Women
}

\author{
Joo-yeong Eom ${ }^{1}$,Sung-je $\mathrm{Cho}^{2}$ \\ ${ }^{1} 2838$ Dept. Department of Regional and Welfare Administration Department, \\ Dongbang Culture Graduate Univ., 28 Sungbok-Ro, Sungbokdong, Seoul, Korea \\ ${ }^{2}$ (Corresponding Author) 02838 Dept. Department of Regional and Welfare \\ Administration Department, Dongbang Culture Graduate Univ., 28 Sungbok-Ro, \\ Sungbokdong, Seoul, Korea \\ 2chosj715@daum.net
}

\begin{abstract}
The purpose of this study is to understand the influence of mindfulness and positive psychological capital on psychological burnout of middle-aged women. The study was conducted on 125 middle-aged women who live in Busan Metropolitan City. Frequency analysis, reliability analysis, and correlation analysis were performed using the SPSS program, and regression analysis was performed to investigate causal relationship between the variables. The study found that first, the mindfulness factor recognized by middle-aged women has a significant influence on psychological burnout. Second, among the subfactors of positive psychological capital perceived by middle-aged women, only the hope factor has an influence on psychological burnout. On the other hand, other subfactors of positive psychological capital, self-efficacy, optimism and resilience, did not have significant effect on psychological burnout. This means that the more the middle-aged women recognize mindfulness, positive psychological capital, and hope factors, the lower their psychological burnout are. The results of this research are expected to become the basis for devising measures to improve the system for the reduction of psychological burnout among middle-aged women.
\end{abstract}

Keywords: A Mindfulness, Positive Psychological Capital, Hope, Psychological Burnout.

\section{Introduction}

\subsection{Background}

The rapidly changing economic and social environments that cause employment issues, economic weakness, interpersonal problems, and excessive competition, are threatening the mental and psychological health of the modern people. The need for counseling is also increasing for the people who are suffering from mental health problems. Counselors or psychotherapists provide psychological training to patients in terms of emotional, social, and developmental aspects, help prevent maladjustment, and maintain psychological health[1]. Many researches have been conducted to understand the effects of psychotherapy on modern people.

Article history:

Received (January 19, 2019), Review Result (March 24, 2019), Accepted (May 29, 2019) 
The psychotherapists have the most impact when it comes to the treatment of mental health problems. They exhibit "altruistic characteristic," which may be a unique strength in the counseling profession. However, a lot of the psychotherapists are under job stress as they are the ones having to provide care constantly. One of the most studied job-related stresses of the counselors is the psychological burnout. Psychological burnout is defined as the mental, emotional, and physical exhaustion that may result from long-term job-related stresses and inability to recover[2]

The biggest problem about psychological burnout is that once it is perceived, it is only going to get worse. As the psychological burnout progressively develops and becomes progressively worse, counselors are constantly exposed to excessive stress and experience anxiety, nervousness, and fatigue[3]. This can have a long-term negative impact on the counselor's own mental and psychological well-being as well as relationship with the patients. In particular, less experienced counselors, who have a lot of enthusiasm and expectations for their role as counselors but lack in experience and self-confidence, may experience psychological burnout more easily than more experienced counselors[4]. It was found that psychological burnout of less experienced counselors increased as the level of attachment anxiety and attachment avoidance increased. In other words, the more the insecure attachment of less-experienced counselor was, the more he or she experienced psychological burnout. In particular, if the counselor is not experienced and has insecure attachment problem, the difficulties and frustration from counseling may lead the counselor to believe that he/she does not deserve support and love from the patient. When the desire to be accepted and validated is not satisfied, the counselor could experience emotional burnout and impersonation, resulting in psychological burnout[5]. The study of I.H. Baek in 2018 found a significant negative correlation between mindfulness and psychological burnout, and that the "grit" of the less experienced counselor has a positive effect on mindfulness. In other words, the higher the grit level of counselors, the higher the level of mindfulness. This also shows that the mindfulness of the less experience counselors has a negative influence on psychological burnout. These findings suggest that the mindfulness of counselors has influence on psychological burnout and grit, or tendency to sustain interest in and effort toward very long-term goals. It is also considered that the patients are affected by the counselor's grit[6]. Therefore, understanding the effect of mindfulness on psychological burnout of prospective counselors, identifying the interaction between variables, and exploring how psychological burnout of prospective counselors who participate in the psychological counseling training change can greatly help set the direction of psychotherapy education and policy decisions.

The results of previous studies have raised the need for research on mindfulness and psychological burnout of prospective counselors. Some studies in the past have been conducted on psychotherapy and counseling, but not many studies have been conducted on the psychological burnout of prospective psychotherapists. Therefore, this study is intended to provide data on the relationship between mindfulness, psychological capital, and psychological burnout of prospective psychotherapists, in order to help prevent psychological anxiety and stress of patients.

\subsection{Objective}

The purpose of this study is to analyze the influence of mindfulness and positive psychological capital of middle-aged women on their psychological burnout. Specific research questions are as follows:

First, what is the effect of mindfulness of middle-aged women on psychological burnout? 
Second, what is the effect of positive psychological capital of middle-aged women on psychological burnout?

\section{Research Method}

\subsection{Research Tool}

The tools used in this study consisted of three questions about demographic characteristics, 20 questions about mindfulness, 24 questions about positive psychological capital, and 22 questions about psychological well-being. The demographics-related questions included questions about gender, age, and monthly income level. The measuring tool used by J.E. Shim[7] for measuring mindfulness of middle-aged women consisted of 24 questions and each question was measured on a 5-point Likert scale. The measuring tool for positive psychological capital used by B.J. Kim[8] included 6 questions about self-efficacy, 6 questions about hope, 6 questions about optimism, and 6 questions about resilience. These 24 questions measured positive psychological capital of middle-aged women on a five-point Likert scale. Lastly, the tool used by G. S. Kim[9] for measuring the psychological burnout of middle-aged women consisted of 22 questions and each question was measured on a five-point Likert scale.

\subsection{Reliability}

The reliability of each variable used in this study came out to be high, with that of mindfulness at .913, positive psychology capital at .966, and psychological burnout at 899 . Each measuring tool showed high reliability level above the minimum Cronbach's $\alpha$ value of 0.60 .

\subsection{Data Processing and Analysis Method}

The processed data collected in the study was verified at a significance level of $5 \%$ using the SPSS 22.0 program, and the reliability of each research tool was analyzed using a coefficient of Cronbach's $\alpha$. For demographic characteristics of middle-aged women, frequency analysis was conducted. Correlation analysis was performed on the mindfulness, positive psychological capital, and psychological burnout, and regression analysis was performed to analyze the impact relationship.

\section{Results}

\subsection{Demographic Characteristics}

A total of 125 middle-aged women (100\%) were studied in this research, among which 73 (58.4\%) were in their 40s, 47 were in their 50s (37.6\%), and five were between age of 60 and 65 (4.0\%). The monthly income level of 59 people (47.2\%) was between 3 and 4 million Won, followed by 34 people (27.2 \%) between 4 and 5 million Won, 27 people (21.6 \%) between 2 and 3 million Won, and 5 people (4.0\%) at 5 million Won or more

\subsection{Statistical Analysis on Main Variables}

The main variables of middle-aged women were measured on a five-point scale. By major factors, mindfulness $(\mathrm{M}=4.12)$ had the highest score and positive psychological capital 
$(\mathrm{M}=4.05)$ scored the second highest. Both factors were above the average. The psychological burnout $(\mathrm{M}=1.92)$ factor had lower than the average score.

\subsection{Correlation between Variables}

Examining the association between mindfulness, positive psychological capital, and psychological burnout of middle-aged women, it was found that there is a high level of positive correlation between mindfulness and positive psychological capital $(\mathrm{r}=.623, \mathrm{p}<.01)$. On the other hand, there was a high level of negative correlation between mindfulness and psychological burnout $(\mathrm{r}=-.805, \mathrm{p}<.01)$. Positive psychological capital and psychological burnout factors were also found to be in somewhat high level of negative correlation $(r=.660$, $\mathrm{p}<.01$ ). The results showed that the correlation coefficient of each variable was correlated at a statistically significant level.

Table 1. Correlation between Main Variables

\begin{tabular}{|c|c|c|c|}
\hline Category & Mindfulness & $\begin{array}{c}\text { Positive Psychological } \\
\text { Capital }\end{array}$ & Psychological Burnout \\
\hline Mindfulness & 1 & & \\
\hline $\begin{array}{c}\text { Positive Psychological } \\
\text { Capital }\end{array}$ & $.623 * *$ & 1 & \\
\hline Psychological Burnout & $-.805^{* *}$ & $-.660 * *$ & 1 \\
\hline
\end{tabular}

${ }^{*} \mathrm{p}<.05, * * \mathrm{p}<.01$

\subsection{Effect of Mindfulness and Positive Psychological Capital of Middle-Aged Women on Psychological Burnout}

\subsubsection{Effect of Mindfulness of Middle-Aged Women on Psychological Burnout}

Regression analysis was performed to investigate the effect of mindfulness perceived by middle-aged women on psychological burnout. It was found that the higher the mindfulness of middle-aged women, the lower her psychological burnout.

Table 2. Effect of Mindfulness of Middle-Aged Women on Psychological Burnout

\begin{tabular}{|c|c|c|c|c|c|c|}
\hline \multirow{2}{*}{$\begin{array}{c}\text { Dependent } \\
\text { Variable }\end{array}$} & \multirow{2}{*}{$\begin{array}{l}\text { Independent } \\
\text { Variable }\end{array}$} & \multicolumn{2}{|c|}{ Unstandardized Coefficient } & \multirow{2}{*}{$\begin{array}{c}\begin{array}{c}\text { Standardized } \\
\text { Coefficient }\end{array} \\
\beta\end{array}$} & \multirow{2}{*}{$\mathrm{t}$} & \multirow{2}{*}{$\begin{array}{c}\text { Significanc } \\
\text { e Level }\end{array}$} \\
\hline & & B & $\begin{array}{l}\text { Standard } \\
\text { Deviation }\end{array}$ & & & \\
\hline \multirow{2}{*}{$\begin{array}{c}\text { Psychological } \\
\text { Burnout }\end{array}$} & Constant & 4.918 & .201 & & $24.501 * * *$ & .000 \\
\hline & Mindfulness & -.725 & .048 & -.805 & $-14.993 * * *$ & .000 \\
\hline
\end{tabular}


$\mathrm{R}^{2}=.648$, Adjusted $\mathrm{R}^{2}=.645$

$\mathrm{F}=224.779, \mathrm{P}=.000$

${ }^{*} \mathrm{p}<.05,{ }^{* *} \mathrm{p}<.01,{ }^{* * *} \mathrm{p}<.001$

\subsubsection{Effect of Positive Psychological Capital of Middle-Aged Women on Psychological Burn-Out}

Multiple regression analysis was conducted to find out the effect of perceived positive psychological capital on psychological burnout of middle-aged women. The R2 value, which indicates the explanation power of positive psychological capital for psychological burnout, was found to be .456 , hence, the regression model can be said to describe $45.6 \%$ of the total variance. In addition, the D-W (Durbin Watson) value of 1.578, which is not close to zero or four, indicates that there was no autocorrelation. Having the tolerance limit higher than 0.1 and the VIF less than 10, no multicollinearity is observed. From the ANOVA, the estimated regression model was found to be statistically significant $(\mathrm{F}=24.909, \mathrm{p}<.000)$. Among the subfactors of positive psychological capital, in terms of their influence on burnout, hope factor ( $\beta=-.475, \mathrm{p}<.01)$ was found to have a significant negative influence. Other subfactors of positive psychological capital, self-efficacy $(\beta=-.210, \mathrm{p}>.05)$, optimism $(\beta=.116, \mathrm{p}>.05)$, and resilience $(\beta=-.119, p>05)$, were found to not have significant influence on psychological burnout. This means that the higher the hope factor perceived by the middle-aged women, the lower the psychological burnout.

\section{Table 3. Effect of Positive Psychological Capital of Middle-Aged Women on} Psychological Burnout

\begin{tabular}{|c|c|c|c|c|c|c|}
\hline \multirow{2}{*}{$\begin{array}{l}\text { Dependent } \\
\text { Variable }\end{array}$} & \multirow{2}{*}{$\begin{array}{l}\text { Independent } \\
\text { Variable } \\
\text { (Positive } \\
\text { Psychological } \\
\text { Capital) } \\
\end{array}$} & \multicolumn{2}{|c|}{ Unstandardized Coefficient } & \multirow{2}{*}{$\begin{array}{c}\text { Standardized } \\
\text { Coefficient } \\
\beta\end{array}$} & \multirow{2}{*}{$\mathrm{t}$} & \multirow{2}{*}{$\begin{array}{c}\text { Tolerance } \\
\text { Limit }\end{array}$} \\
\hline & & B & $\begin{array}{l}\text { Standard } \\
\text { Deviation }\end{array}$ & & & \\
\hline \multirow{5}{*}{$\begin{array}{l}\text { Psychological } \\
\text { Burnout }\end{array}$} & Constant & 3.851 & .211 & & $18.231^{* * *}$ & \\
\hline & Self-Efficacy & -.138 & .164 & -.210 & -.844 & .074 \\
\hline & Hоре & -.325 & .119 & -.475 & $-2.722 * *$ & .150 \\
\hline & Optimism & .077 & .124 & .116 & .621 & .132 \\
\hline & Regre & -.088 & .145 & -.119 & -.606 & .119 \\
\hline \multicolumn{7}{|c|}{$\mathrm{R}^{2}=.456$, Adjusted $\mathrm{R}^{2}=.437$} \\
\hline \multicolumn{7}{|c|}{$\mathrm{F}=24.909, \mathrm{P}=.000$, Durbin-Watson=1.578 } \\
\hline
\end{tabular}

${ }^{*} \mathrm{p}<.05, * * \mathrm{p}<.01, * * * \mathrm{p}<.001$

\section{Conclusion and Discussion}

This study analyzed the effects of perceived mindfulness and positive psychological capital on psychological burnout of middle-aged women. Based on the results of the analysis, the details are discussed as follows: 
First, the factors of mindfulness perceived by middle-aged women showed significant influence on psychological burnout.

Second, among the subfactors of positive psychological capital perceived by middle-aged women, only the hope factor influenced psychological burnout.

As discussed above, the need for positive psychological capital is increasing as it can help reduce psychological burnout of middle-aged women. Therefore, the present study has significance in that it analyzes psychological burnout related to mindfulness and positive psychological capital of middle-aged women, when the existing studies were more about the effects of psychological counseling and case studies of middle-aged women. Based on these findings, it seems necessary to develop and conduct customized education programs that can address psychological burnout of middle-aged women.

\section{References}

[1] E. J. Yun, "An Experiential Analysis of Burnout in Counselors", Sookmyung Women's University Graduate School. (2007)

[2] Yean-Hong. Jeong, "A Study for the Development of the Teacher Burnout Inventory(TBI)", Korea National University of Education Chung-Buk Major in Counseling Psychology Graduate School, Doctoral thesis. (2016)

[3] S. H. PARK, "Influence of Occupational Stress and Social Support on Counselors Burnout", The graduate school of Ewha Womans University, Master's Thesis. (2001)

[4] M. J. Kim. "Development of Scale about Novice Therapists' Difficulty Experienced in Counseling”, The graduate school of Catholic University, Master's Thesis. (2006)

[5] Hey soo Lee, "The Relation Between Adult Attachment and Burnout of Novice Counselor: The Mediation Effects of Basic Psychological Needs Satisfaction", The Catholic University of Korea. Master's Thesis. (2011)

[6] In-Hye Baek, " The relationship between novice counselor`s GRIT and burnout : the mediation effects of mindfulness", The graduate school of Catholic University(2018), Master's Thesis. (2018)

[7] J. E. Shim, "Counselor's Mindfulness, Empathy, and Countertransference Management Ability.", Department of Psychology Graduate School The Catholic University of Korea. J.E. Shim - Mindfulness Scale. (2008)

[8] B. J. Kim, "The Influence of Positive Psychological Capital to the Job Satisfaction of Child Care Teachers Verification of the Mediating Effect of Psychological Exhaustion", Department of Social Welfare Graduate School Woosuk University. B.J. Kim - Positive Psychological Capital Scale. (2018)

[9] G. S. Kim, "The impact of Counselor's Self-reflection on the Psychological Burnout.", Department of Psychology Graduate School the Catholic University of Korea. (2013)

\section{Authors}

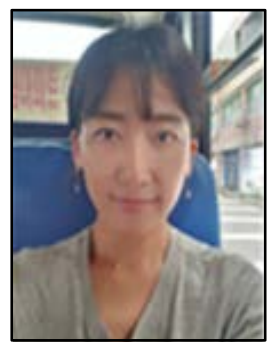

Joo-yeong Eom

$2018 \sim$ present

Team Leader of Big Dream Contents Institute of Korea Counseling Education Center 